\title{
Response of a Biphasic Poly(n-hexyl isocyanate)/p-Xylene Solution to Applied High Electric Fields
}

\author{
TODD J. MENNA, FRANK E. FILISKO \\ Materials Science and Engineering, University of Michigan, Ann Arbor, Michigan 48109
}

Received 14 November 2003; revised 9 December 2004; accepted 16 December 2004

DOI: 10.1002 / polb.20400

Published online in Wiley InterScience (www.interscience.wiley.com).

\begin{abstract}
The effect of a high electric field on a solution of a lyotropic liquid-crystalline polymer, $\operatorname{poly}(n$-hexyl isocyanate) in $p$-xylene, was studied. The application of a high-voltage alternating-current electric field to the biphasic solution resulted in an elongation of the nematic domains in the field direction, the degree of elongation varying approximately with the square of the electric field. At a constant field, the extent of elongation decreased, varying inversely with the frequency in an exponential fashion. The domain structure and thus the molecular orientation were examined to explain these electric field effects. (C) 2005 Wiley Periodicals, Inc. J Polym Sci Part B: Polym Phys 43: 1124-1133, 2005
\end{abstract}

Keywords: liquid-crystalline polymers (LCP); poly(n-hexyl isocyanate) (PHIC); rheology; structure-property relations

\section{INTRODUCTION}

It is well known that the application of electric and magnetic fields to appropriate materials can induce dramatic changes in various physical, optical, and mechanical properties. For example, the flow properties of certain dispersions can change by many orders of magnitude when the dispersions are exposed to an electric field. This effect is associated with the electric field (and shear field) induced aggregation of the particles in specific patterns. ${ }^{1}$ Significant rheological changes have also been reported for certain lyotropic liquid-crystalline (LC) systems, ${ }^{2,3}$ the explanation of which cannot only involve particle association because the solutions are homogeneous; that is, the particles do not exist.

Reports of the study of this electrorheological (ER) effect go back to the $1890 \mathrm{~s},{ }^{4}$ with major emphasis being put on the study since the 1940s with the investigations by Winslow ${ }^{5}$ on particle dis-

Correspondence to: T. J. Menna (E-mail: tmenna@engin. umich.edu)

Journal of Polymer Science: Part B: Polymer Physics, Vol. 43, 1124-1133 (2005) (C) 2005 Wiley Periodicals, Inc. persion ER fluids. In the 1960s, LC materials began to be readily available, and investigations into their ER effects soon followed, but they lagged behind particulate suspensions. The consideration of LC materials in these applications expanded in the mid-1980s because of the advantages of LC solutions over particulate suspensions, which include the homogeneous nature of the fluid, decreased abrasion, and absence of the settling effect.

To date, numerous studies have created an abundant number of theories and hypotheses concerning the behavior of LC solutions in response to the application of an electric field, shear, or both. Most notable are the theories of Ericksen, Leslie, and Parodi (ELP), ${ }^{6}$ Brochard, ${ }^{7}$ and Doi. ${ }^{8}$ However, the ELP theory is limited to low flow rates, and the material must be highly characterized by other methods, whereas the theories of Brochard and Doi, although superior in their explanations regarding the shear response of liquid crystals, are not entirely acceptable because of their failure to incorporate the effects of imposed electric fields. Thus far, the most successful theoretical considerations have come from Tse and Shine, ${ }^{9}$ who used a two-dimensional version 
of the Doi theory developed by Maffettone and Marrucci, ${ }^{10}$ yet still no experimental theory or modeling process has successfully included all of the variables (shear rate, molecular species, temperature, concentration, field type, voltage, frequency, etc.), and so no uniform, complete theory has been developed.

A majority of researchers to date have primarily focused on the rheological behaviors of an LC solution in response to high electric fields. However, very little work has been done to characterize the morphological responses of a lyotropic LC material under an electric field, and this is important for understanding the ER properties. ER studies have also tended to focus on just one phase, that is, the nematic phase, with either rigid-rod polymers ${ }^{2}$ or side-chain liquid crystals. ${ }^{11,12}$ Gleeson et al. ${ }^{13}$ and Larson and $\mathrm{Mead}^{14}$ put forth a very thorough explanation of the morphological response during the slow shearing of LC solutions of poly(benzyl glutamate) enantiomorphs and poly( $\gamma$-benzyl-L-glutamate) solutions, respectively, yet their work did not incorporate the use of an electric field.

In an attempt to help explain these ER effects and other behaviors, we explored morphologically the behavior of a lyotropic LC system under various conditions. The effects of a high electric field, frequency, and shear rate on biphasic concentrations, those consisting of nematic domains in an isotropic matrix, of a well-known and accepted LC polymer were investigated to achieve this goal.

This research involved the observation of the morphological responses of a lyotropic LC polymer, $\operatorname{poly}(n$-hexyl isocyanate) (PHIC), in solution with $p$-xylene to applied high electric fields. This system is uniquely applicable because it is the only LC polymer that can be dissolved in a very poor electrically conducting solvent in large quantities (40 wt \%) without the formation of a highly viscous solution or gel.

\section{EXPERIMENTAL}

\section{Polymer Acquisition}

PHIC was synthesized by our group in a manner similar to that of Shashoua et al. ${ }^{15}$ and Aharoni. ${ }^{16}$ The synthesis method is described in another publication ${ }^{17}$ and provides, through its methodology, higher yields and better control over the polydispersity. PHIC had a weight-

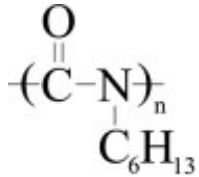

Figure 1. Repeat unit of PHIC.

average molecular weight of $50,000 \mathrm{~g} / \mathrm{mol}$, as determined from the intrinsic viscosity measurements $\left(92.7 \mathrm{~g} / \mathrm{cm}^{3}\right.$; viscosity-average molecular weight $=$ $22,600 \mathrm{~g} / \mathrm{mol}$ ) with the equation of Bur and Fetters, ${ }^{18}$ and a weight-average degree of polymerization of 394. Figure 1 shows the repeat unit of the PHIC molecule. The solution used in this study was of PHIC dissolved in $p$-xylene (Acros Organics) to a biphasic concentration of $29 \mathrm{wt} \%$.

\section{Equipment}

The solution was placed between parallel $80-\mu \mathrm{m}$ copper-foil electrodes (1.1-mm gap) and was observed at a magnification of $50 \times$ with a Nikon Diaphot 200 inverted stage microscope. The microscope was mounted on an $x-y$ translational stage capable of movement throughout the entire sample region. Because the observation cell stage and the microscope were not coupled, the $x-y$ translational stage allowed the microscope to be smoothly moved independently throughout the observation cell area, and the observation cell area was left free of any external interference. Alternating-current electric fields were applied across the electrode gap with a Trek 664 high-voltage power supply coupled with a Kikusui 455 function generator for frequency applications.

A Pulnix TMC-7 color camera was adapted to the top of the microscope and attached to a video recording device for future reference to the conducted experiments. Digital photographs were taken with a Dazzle Digital Photo Maker DM-5000 device and software attached to a desktop personal computer. The samples were observed under cross-polarized light with the polarizer and analyzer oriented parallel and perpendicularly, respectively, to the direction of the electric field. The polarizer and analyzer were held stationary throughout all the testing. All the testing was done at $23{ }^{\circ} \mathrm{C}$.

\section{Morphology}

An alternating-current electric field was placed over a biphasic solution of PHIC in $p$-xylene. 

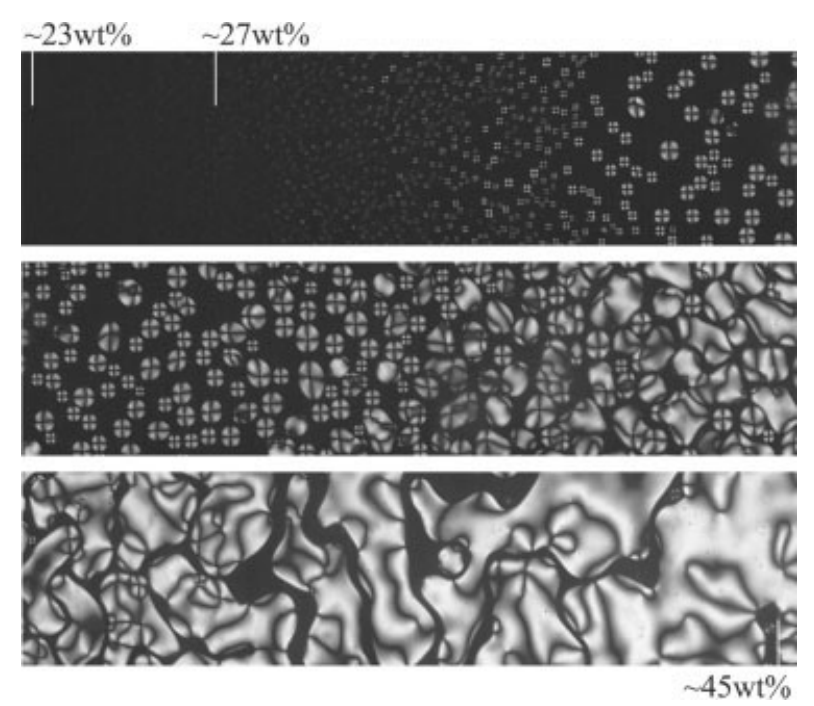

Figure 2. Concentration gradient for a $\mathrm{PHIC} / p$-xylene solution showing the existence of the isotropic, biphasic, and nematic phase domains between cross-polarizers oriented horizontally and vertically to the image.

The response was recorded visually by the recording of the morphological changes in the nematic domains within the isotropic matrix. The applied field $(\mathrm{V} / \mathrm{mm})$ was increased until no measurable dimensional changes were observed, or orientation caused total extinction, and then reduced incrementally back to zero. The measurements were made with both increasing and decreasing alternating-current field strengths at frequencies of 100, 400,700, 1000, and $2000 \mathrm{~Hz}$. Planimeter data were acquired with a K\&E 620000 compensating polar planimeter.

\section{RESULTS}

\section{PHIC Concentration Morphology}

Figure 2 shows the concentration gradient (divided into three images) for the $\mathrm{PHIC} / p$-xylene system from 23 to $45 \mathrm{wt} \%$ PHIC under a zero electric field. The images were obtained though the controlled evaporation of a $23 \mathrm{wt} \%$ solution, and the concentration increases from left to right and from top to bottom. The labeled concentrations are approximations determined from known concentration images. All three phase domainsisotropic $(<27 \mathrm{wt} \%)$, biphasic $(27-45 \mathrm{wt} \%)$, and nematic ( $>45 \mathrm{wt} \%)$-are shown. The isotropic-tonematic phase-transition point is evident in the formation of discrete, circular nematic domains, which appear at approximately $27 \mathrm{wt} \%$. These domains increase in size with increasing concentration, impinging upon and coalescing with one another as neighboring domains attempt to occupy the same space. Disclination lines indicating radial symmetry are found within the nematic domains, and as the domains coalesce and grow, they show a loss of symmetry and the development of a fully nematic structure. The coalescence is indicative of the onset of the biphasic-nematic transition concentration of approximately $35 \mathrm{wt} \%$, which is evident in the middle of the central image of Figure 2.

Initially, the disklike nematic domains contain one point disclination at their center with four radial brushes emanating from this point; this is characteristic of a nematic structure. However, with increasing concentration, the impingement of the nematic domains occurs, and the brushes distort as the symmetry of the molecular orientation changes because of coalescence of the material between the two domains. Increasing the concentration increases the size of the coalesced domains such that the isotropic phase now becomes the minority phase in a matrix of large nematic islands. These islands in turn grow and coalesce with one another, creating the final $100 \%$ nematic structure (not shown in Fig. 2). With increasing coalescence, single domain orientation is lost, and the resultant nematic structure shows evidence of both one and two brush point disclinations, as expected for a nematic structure.

\section{Domain Deformation}

The work in this study is focused on the response of a nematic domain in the biphasic region, which is shown in the right two-thirds in the top image of Figure 2, when it is subjected to an electric field. As shown in Figure 3 the domain is transformed from disklike to elliptical, the major axes being in the field direction, with a concurrent increase in the field strength. This elongational effect is reversible with a decrease in the electric field to the point at which, if enough time is allowed, the nematic domain will return to its original dimensions. Although Figure 3 focuses on only one domain, the same effects occur in all domains, both smaller and larger, as noted within the figure, with the smaller domains on the outskirts of the images and at other frequencies as well. The figure is merely a representation of the effects of the field and frequency, focusing on one specific 

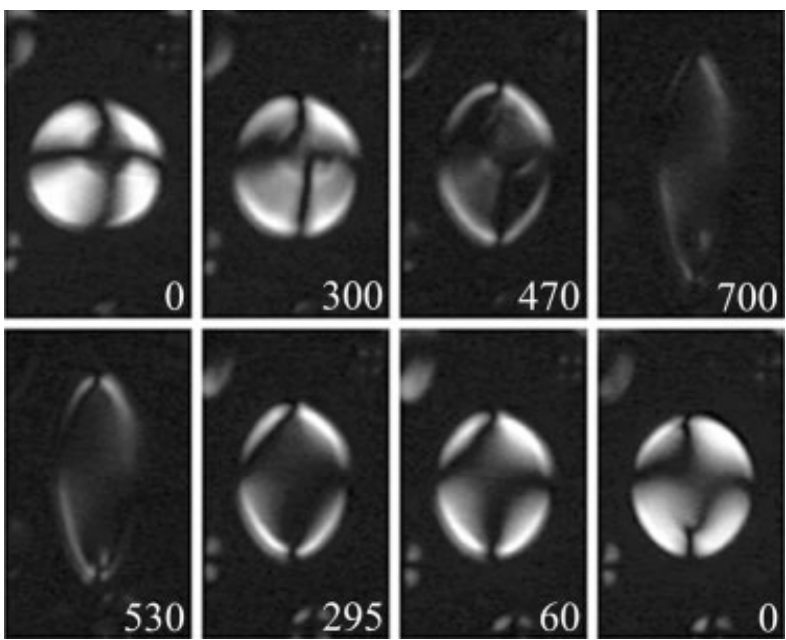

Figure 3. Nematic domain orientation and extension in the field direction. The numbers refer to volts per millimeter. The field was in the vertical direction. The frequency was $100 \mathrm{~Hz}$. The polarizer and analyzer were oriented vertically and horizontally, respectively. One image cell is approximately $60 \mu \mathrm{m}$ wide.

domain that occurs for numerous concentrations on numerous occasions. The elongational effect is very similar to that shown to occur for immiscible polymer blends ${ }^{19}$ and the coalescence of elongated domains is possible; in turn, they occasionally elongate even to the point of completely bridging the gap between electrodes. The domains continue to elongate in the field direction at fields greater than the $700 \mathrm{~V} / \mathrm{mm}$ shown here. However, the gain of retardancy of the sample with an increase in the field eventually decreases to a point at which the nematic domain is very difficult to distinguish from the surrounding isotropic matrix. The domain is still visible at $700 \mathrm{~V} / \mathrm{mm}$, and thus for imaging purposes, imaging stops at this point.

Perhaps the most significant observation from Figure 3 is the change in the structure of the disclination at the center of the domain as the electric field is increased and decreased. The disclination begins as the typical Maltese cross pattern characteristic of radial symmetry, such as that found in polymer spherulites, yet upon the application of the electric field, the disclination, originally one central point, breaks into four distinct line disclinations that move toward their corresponding corner with an increase in the electric field strength. The slight lack of symmetry in the photographs here is an experimental artifact brought about by the poor alignment of the polarizer and analyzer, which are completely

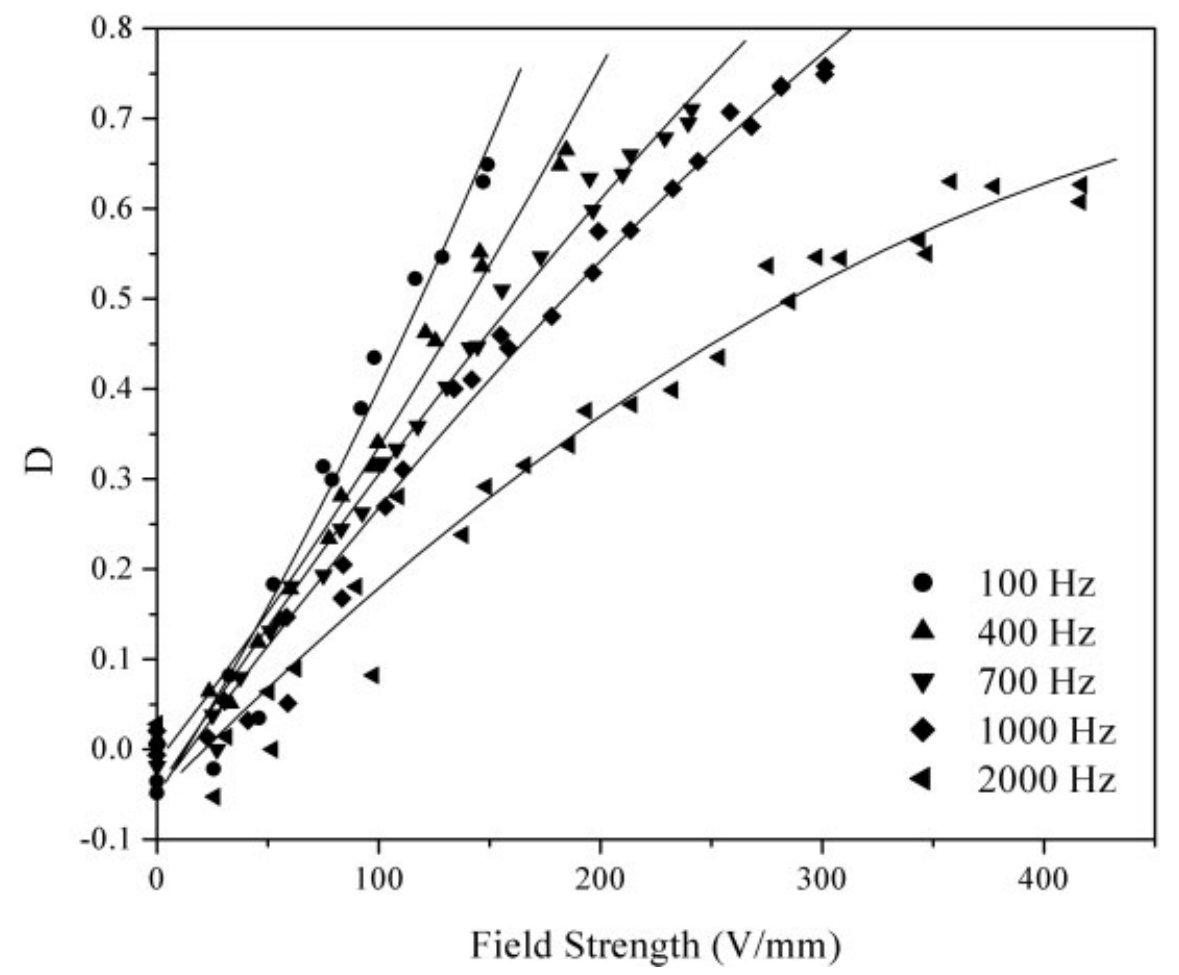

Figure 4. Deformation of a nematic domain with an applied electric field. The points were obtained while the electric field increased and while it decreased. 


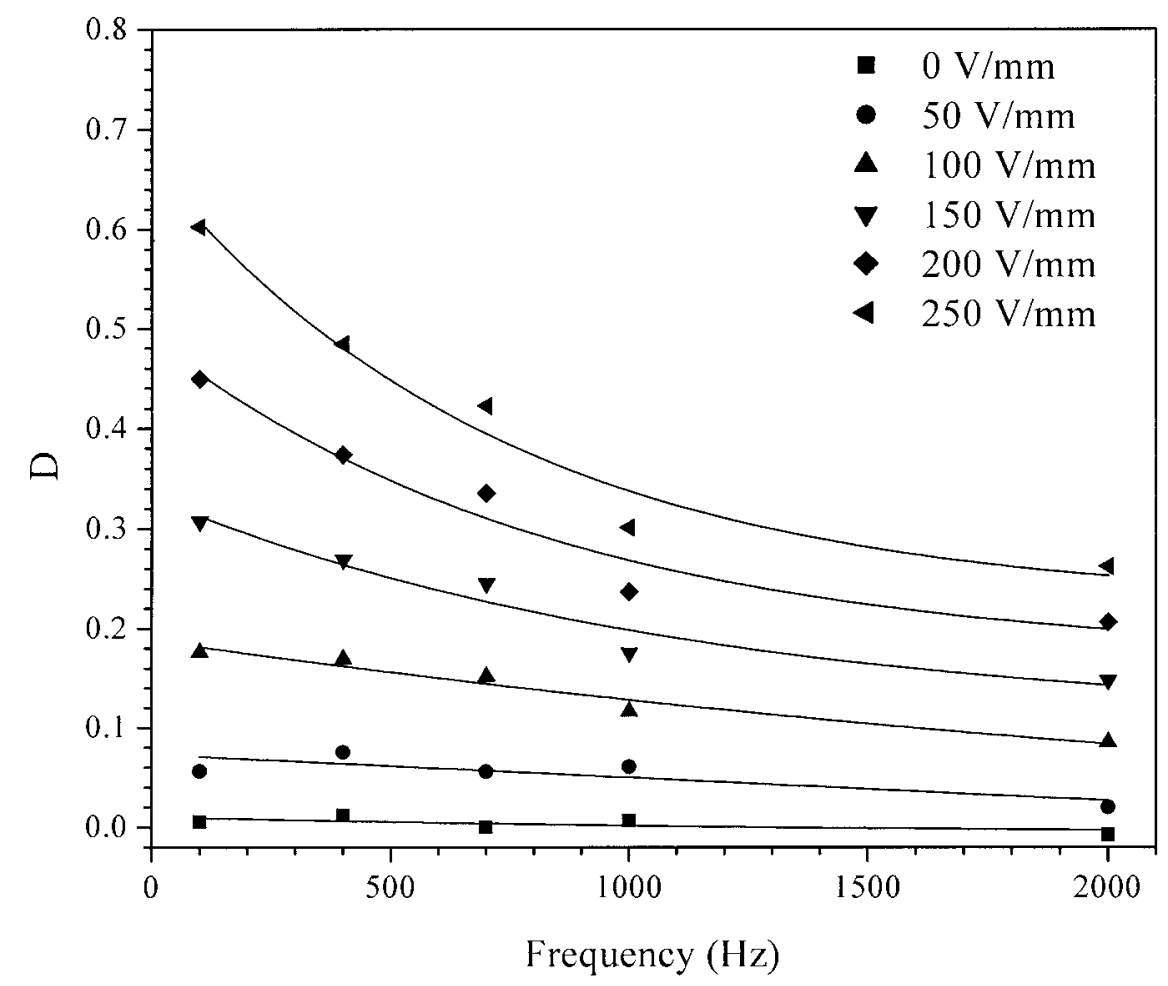

Figure 5. Elongation of a nematic domain decreasing with increasing frequency at a constant field strength. The lines are exponential fits of the actual data.

parallel and perpendicular to the applied electric field. During the disclination migration process, the material remaining within the center of the domain darkens because of orientation of the molecules in the direction of the polarizer. This oriented region originates from the center of the domain and expands to the domain boundaries as the field is increased. The effect is reversible: with a decrease in the electric field strength, the disclinations reform and begin to move toward the center of the domain. Although not shown in the figure, the disclinations will meet at the center, and the overall Maltese cross pattern will reform. For this sample, approximately 30 s was allowed for the sample relaxation between field changes, yet this pattern re-creation can take from $60 \mathrm{~s}$ to several hours to occur, depending on the initial domain size.

The degree of deformation $(D)$ of an ellipsoidal droplet can be defined as follows: ${ }^{20}$

$$
D=\frac{a-b}{a+b}
$$

where $a$ and $b$ represent the lengths of the major and minor axes of the ellipsoid, respectively. In this work, the major and minor axis were taken to be parallel and perpendicular to the electric field direction, respectively, and thus the value of $D$ can range from 0 for a perfect sphere to 1 (ignoring volume conservation, etc.) for an infinite-length rod structure. Figure 4 notes graphically the elongational response in terms of $D$ from samples similar to Figure 3 and shows an increase in the magnitude of $D$ (in the field direction) that increases proportionally to the strength of the alternating-current field. The figure shows both the increasing and decreasing aspect ratio measurements of the same nematic domain, showing that within experimental error the prior deformation of the sample does not affect the domain's stable dimensions at a particular frequency-field combination.

Increasing the applied frequency reduces the magnitude of the increase by which the nematic domain elongates in response to the field. For this particular data set, approximately 30 s was allowed for sample relaxation with decreasing field strength at each frequency. As shown by the data, scatter increases with increasing frequency, and this is related to the longer times needed to allow for relaxation at higher frequencies. 


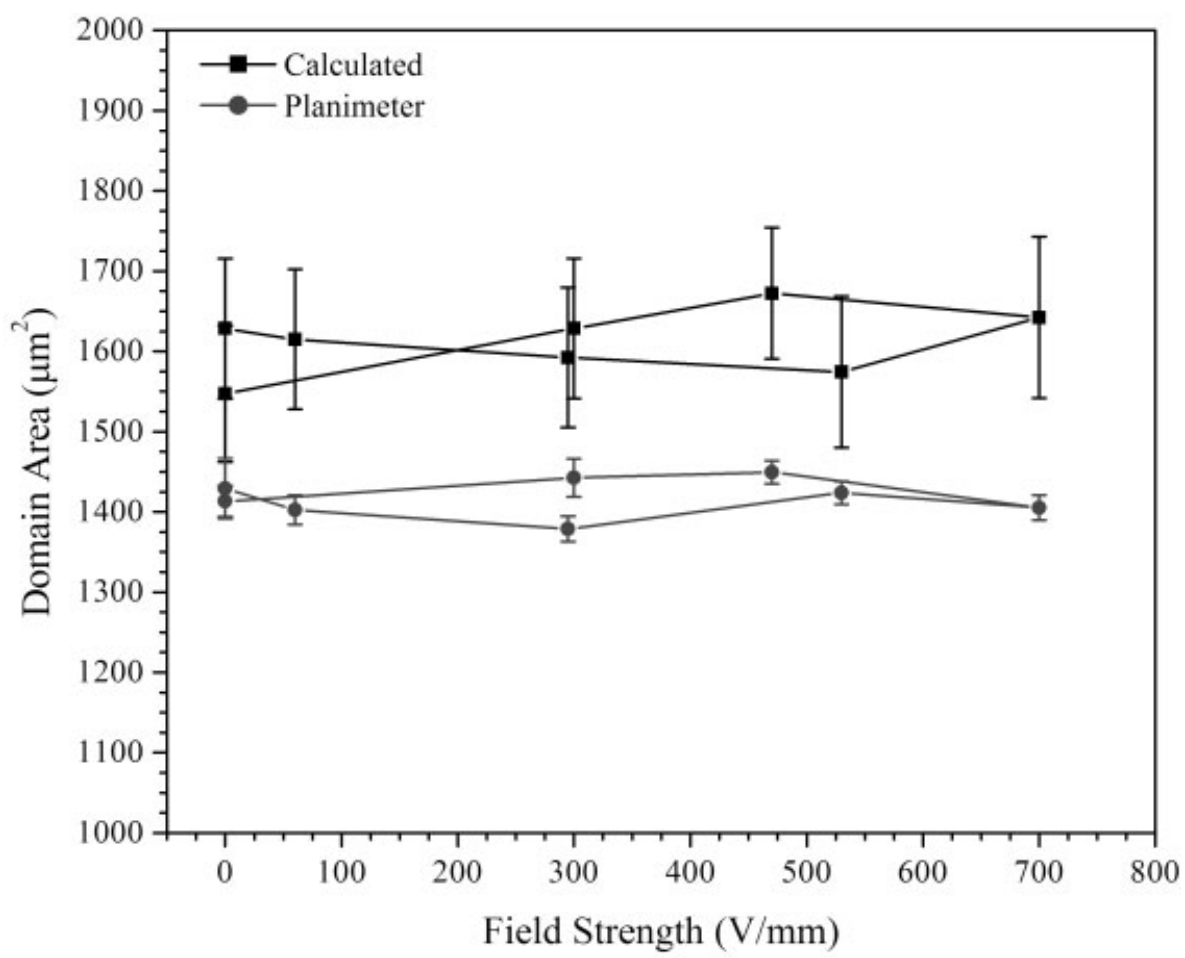

Figure 6. Domain cross-sectional area as a function of the field strength at $100 \mathrm{~Hz}$.

If instead the field is held constant and the frequency is varied (Fig. 5), an elongated nematic domain will shrink; that is, $D$ will decrease with increasing frequency. As noted in Figure 4, a larger electric field is required to achieve a larger value of elongation. However, at sufficiently high frequencies, the domain deformation approaches an asymptotic limit that is dependant on the electric field strength, as shown in Figure 5. The value of $D<0$ is due to an initial slightly oblate shape of the droplet at a zero electric field. The trend produced is accurate, showing no change in the droplet dimension with no applied electric field.

\section{Domain Area}

Figure 6 illustrates how the cross-sectional area of the domains, under the assumption of a transformation from a circular shape to an elliptical shape with increased deformation, changes in response to the increased electric field. Within experimental error, no change in the cross-sectional area of the domain is evident.

Two methods were used in the determination of the cross-sectional area of the domain shown in Figure 3. The first involved mathematically calculating the cross-sectional area under the assumption of a domain shape transformation from circular geometry to elliptical geometry upon extension, whereas the second involved the use of a planimeter device to measure the area directly from the image. As noted in Figure 6 , the two methods produced similar results, with the calculated domain area being slightly larger yet still within a reasonable error of the planimeter data. This plot is significant, in that it shows that with an increase in the electric field, the domain cross-sectional area remains relatively constant; this indicates that the elliptical assumption is satisfactory. Using this, we can extrapolate the assumption into a prediction of a spherical domain structure.

\section{Domain Volume}

Regarding the elongation of the nematic domain in the field direction, as the molecules within the domain begin to orient, volume considerations can be taken into account. The molecules cannot occupy the same space and, because of their dipolar attraction to the electric field, will migrate toward the electrodes. Ideally, the volume of the domain should remain consistent during elonga- 


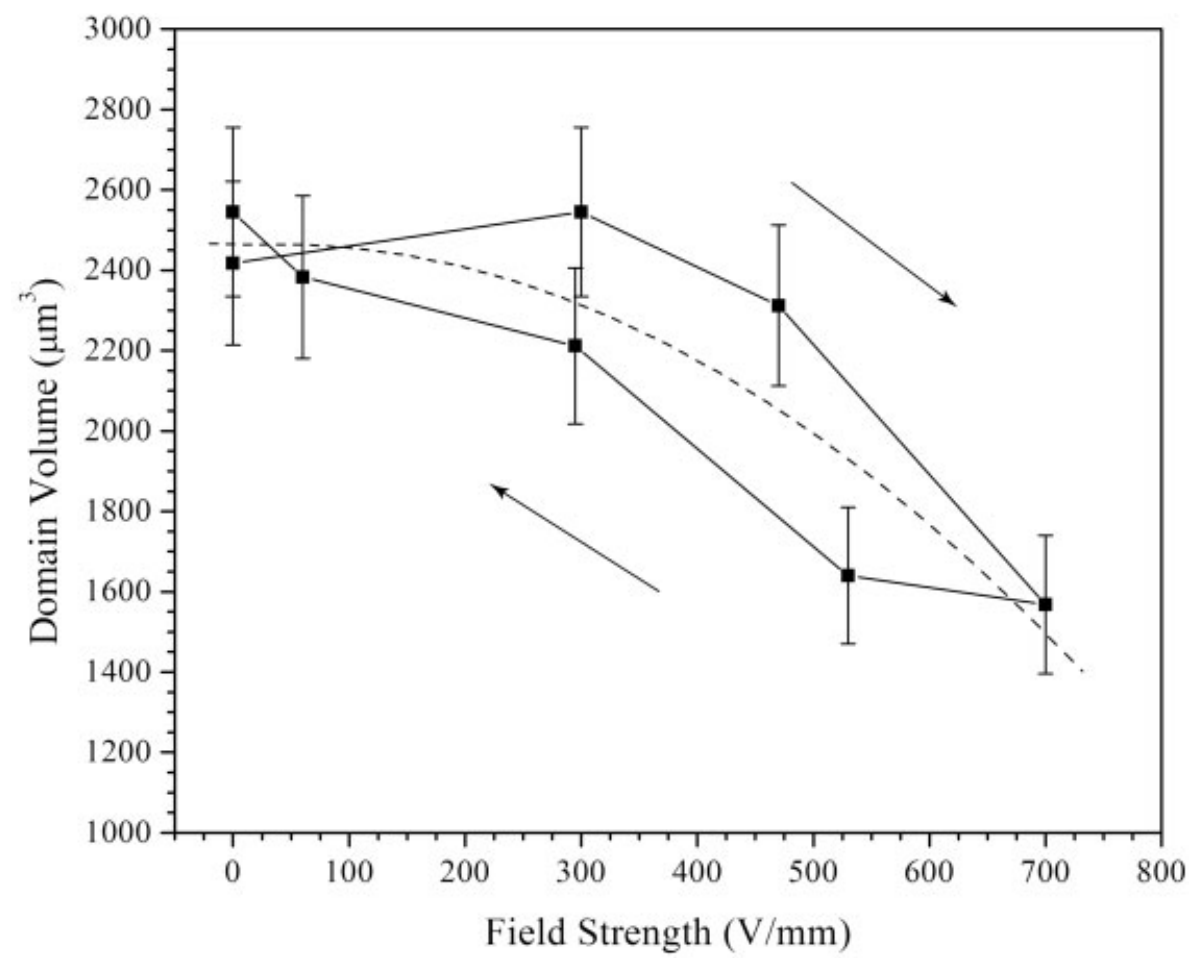

Figure 7. Domain volume analysis for the elongated domain in Figure 3 at $100 \mathrm{~Hz}$. The arrows indicate the direction of movement along the line. The dotted line indicates the average domain volume during the field cycle.

tion, with an increase in the length reducing the width proportionally. However, because of increased molecular alignment, the overall domain density should increase. If the mass within the nematic domain remains constant, the volume of the domain must consequently decrease. Using the same parameters to calculate the volume of the ellipsoid that were used to calculate the areas in Figure 6, we find that this does occur, as demonstrated in Figure 7 for the spherical domain represented in Figure 3. Figure 7 shows that the domain volume decreases with the domain elongation in response to an increasing electric field strength. The dashed line in the figure indicates the average of the increasing and decreasing volume with a changing electric field. Just as the domain returns to its original shape with a decrease in the electric field, the volume returns to its original magnitude with a decrease in the electric field.

Just as an increase in frequency will reduce the deformation of a domain, a frequency change should also change the droplet volume. Using the information from Figure 7 in conjunction with Figure 5, we can obtain the relationship between the domain deformation and domain volume at
$100 \mathrm{~Hz}$. Figure 8 summarizes this relationship at $100 \mathrm{~Hz}$ and indicates that for a given field strength, increasing the frequency reduces the deformation, and this in turn increases the domain volume.

\section{DISCUSSION}

The data from Figures 4 and 5 show that at a particular field-frequency combination, the domain elongates to a particular deformation value and remains constant in shape. Increasing the applied field and keeping the frequency constant increase $D$, and increasing the frequency at a constant field strength effectively reduces $D$. However, because the frequency and field strengths have opposite effects on the deformation, if one variable is changed, the other must change for a constant domain size to be maintained.

As a fundamental property of dielectrics, a uniform electric field will be distorted by the presence of a dielectric sphere, in a manner similar to that shown in Figure 9. The electric field lines will bend toward the sphere, in this case a 


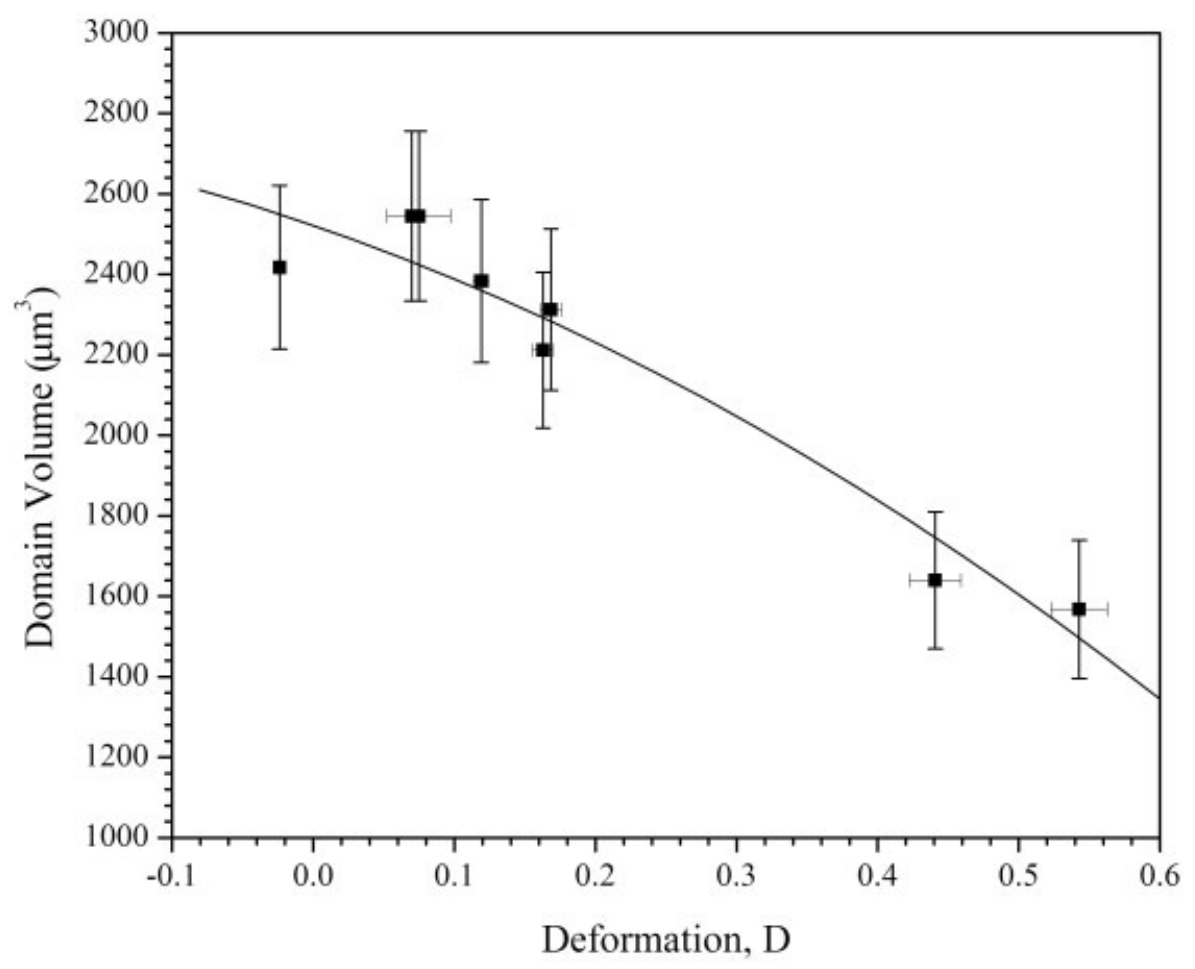

Figure 8. Domain volume as a function of $D$ at $100 \mathrm{~Hz}$.

nematic domain, the angle of bending increasing as the surface tangent approaches parallelism with the applied electric field. However, the interior of the nematic domain sees a continuation of the electric field lines, still parallel to one another, yet at decreased spacing in comparison with that of the isotropic exterior. ${ }^{21}$ Because these domains extend in the direction of the electric field, the dielectric constant within the domain must be greater than that of the surrounding material. The higher permittivity of the domain results in a partial cancellation of the electric field within the domain and is indicated by the dashed field lines within the domain in Figure 9. This phenomenon is symmetrical within the nematic domain in the field direction.

For PHIC/toluene biphasic solutions, at a zero field the molecules within the nematic domain at the interface of the nematic and isotropic phases align parallel to the domain surface. ${ }^{22}$ Because the chemical structures of toluene and $p$-xylene differ only slightly, the assumption can be made that the molecules should behave in a similar manner in our $p$-xylene solutions. Before any field application, Figure 10(a) illustrates the domain molecular structure after the extrapolation of this surface tangent orientation throughout the bulk of the domain. Upon the application of an electric field, the molecules begin to rotate into the field direction, the orientation increasing as the field increases [Fig. 10(b-d)]. Figure 10 represents the orientation of the PHIC molecules within the nematic domain not only as the field is increased but also as the domain elongates in the field direction. In response to an applied electric field, the permanent dipoles of the PHIC molecules line up in the field direction. Regardless of the orien-

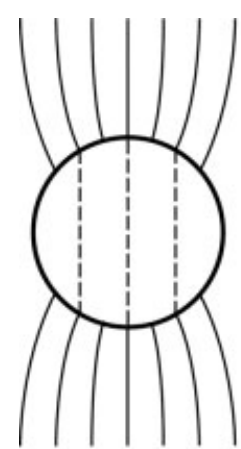

(a)

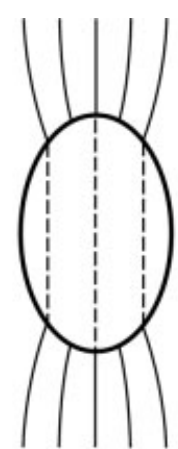

(b)

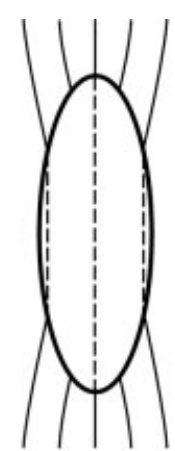

(c)

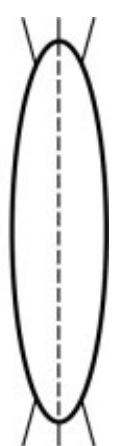

(d)
Figure 9. Field lines through the nematic droplet (a) at an initial low field and (b-d) with increasing field strength. The domain shape change has been taken into consideration. 


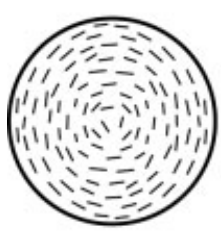

(a)

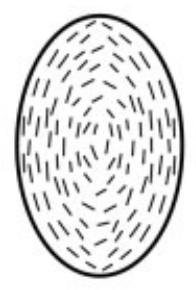

(b)

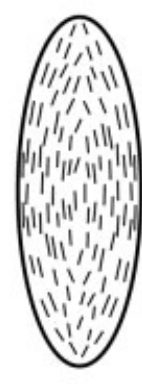

(c)

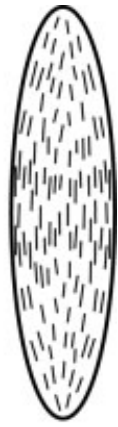

(d)
Figure 10. Response of the PHIC molecules to the applied electric field within the droplet (a) at a zero electric field and (b-d) with increasing field strength.

tation within the domain, orientation increases with increasing electric field strength, and so the molecules along the domain equator have the minimum required rotation and remain parallel to the surface; however, those along the vertical center of the domain have the largest degree of rotation necessary for alignment, $90^{\circ}$. Under cross-polarized light, the resulting retardancy of the domains increases from the domain center outward at a $45^{\circ}$ angle as the field is increased and the domain elongates, as shown in Figure 3.

Thus, when an electric field is applied to a biphasic lyotropic LC solution (consisting of a continuous matrix of a saturated isotropic phase and a dispersed phase of spherical nematic regions), the molecules in the isotropic phase show increased orientation in the field direction proportional to the magnitude of the electric field $E\left(\sim E^{2}\right){ }^{23}$ The molecules in the nematic domains also show an increased orientation in the field direction from their initially radial symmetry at a zero field. This is confirmed by the presence of the Maltese cross pattern on the nematic domain when it is viewed between crossed polarizers under a zero electric field. Additionally, the nematic domains are distorted from their initially circular shape to an apparently elliptical shape, with the major axes extending in the field direction. The degree of extension is proportional to the magnitude of the electric field and inverse to the frequency of the applied alternating field. As the field increases and the elliptical nematic domain becomes longer, the molecular orientation within the domain becomes essentially all in the field direction with the exception of the boundary, which is the last to show retardancy. However, at some field, the nematic domains become com- pletely invisible when they are viewed between crossed polarizers, and this indicates that the PHIC molecules are essentially all oriented in the field direction. Concurrently with the increasing field, it is observed (Fig. 6) that the area of the nematic disks as they distort under the increasing electric field remains essentially the same. However, if it is assumed that the nematic domains are initially spherical ${ }^{24-26}$ and distort as ellipsoids, the volumes calculated from the measured major and minor axes indicate the volume of the domains is decreasing. This may be an artifact, yet it can be shown that for a sphere ellipsoid with a constant cross-sectional area, the volume decreases as the ellipsoid becomes more extended, essentially linearly; this is consistent with our observations and calculations. This suggests two possibilities: (1) the PHIC content of the nematic domain is constant, and so the density must be increasing, and (2) the PHIC molecules at the interface are dissolving from the nematic domain into the surrounding oriented isotropic matrix. The first option is measurable because it would require a very large increase in the density within the nematic domain even with a major/minor axis ratio of $2 / 1$. For instance, a change in the aspect ratio of the nematic domain from 1 to 2 would yield a density increase of $41 \%$, which is highly unlikely. It is more likely that as the volume of the nematic domain decreases, that is, becomes more extended, that PHIC molecules dissolve into the isotropic phase, more so as the field increases and the nematic domains become more extended. Finally, at high enough field strengths, the situation persists, and the extended nematic domain and the oriented isotropic become indistinguishable; that is, there is a single phase (paranematic). ${ }^{27}$ When the field is turned off, numerous nematic domains appear, initially nucleating sporadically in time and growing radially until a situation similar to what was initially observed occurs. However, the state after the field is removed does not always have randomly placed nematic domains. Occasionally, several smaller domains instead of one large domain may nucleate along a line consistent with the previously extended nematic domain. Although no study has been performed for confirmation, it is estimated that the total volume percentage of nematic domains, after sufficient growth time has passed, is relatively equivalent to that present before the application of the electric field. 


\section{REFERENCES AND NOTES}

1. Henley, S.; Filisko, F. E. J Rheol 1999, 43, 13231336.

2. Yang, I. K.; Shine, A. D. J Rheol 1992, 36, 10791103.

3. Inoue, A.; Maniwa, S. J Appl Polym Sci 1996, 59, 797-802.

4. Duff, A. W. Phys Rev Ser 1 1896, 4, 23-38.

5. Winslow, W. M. J Appl Phys 1949, 20, 1137-1140.

6. Carlson, T.; Skarp, K. Mol Cryst Liq Cryst 1981, 17, 157-171.

7. Brochard, F. J Polym Sci Polym Phys Ed 1979, 17, 1367-1374.

8. Doi, M. J Polym Sci Polym Phys Ed 1981, 19, 229-243.

9. Tse, K, Shine, A. D. J Rheol 1995, 39, 1021-1040.

10. Marrucci, G.; Maffettone, P. L. Macromolecules 1989, 22, 4076-4082.

11. Inoue, A.; Maniwa, S. J Appl Polym Sci 1995, 55, 113-118.

12. Yao, N.; Jamieson, A. M. Macromolecules 1997, 30, 5822-5831.

13. Gleeson, J. T.; Larson, R. G.; Mead, D. W.; Kiss, G.; Cladis, P. E. Liq Cryst 1992, 11, 341-364.

14. Larson, R. G.; Mead, D. W. Liq Cryst 1992, 12, $751-768$.
15. Shashoua, V. E.; Sweeny, W.; Tietz, R. J Am Chem Soc 1960, 82, 866-873.

16. Aharoni, S. M. Macromolecules 1979, 12, 94-103.

17. Hopkins, A. R. Masters Thesis, University of Michigan, 1993.

18. Bur, A. J.; Fetters, L. J. Macromolecules 1973, 6, 874-879.

19. Tajiri, K.; Ohta, K.; Nagaya, T.; Orihara, H.; Ishibashi, Y.; Doi, M.; Inoue, A. J Rheol 1997, 41, 335-341.

20. Taylor, G. I. Proc R Soc London Ser A 1934, 146, 501-523.

21. Reitz, J. R.; Milford, F. J. Foundations of Electromagnetic Theory; Addison-Wesley: Reading, MA, 1962.

22. Chen, W.-L.; Sato, T.; Teramoto, A. Macromolecules 1996, 29, 4283-4286.

23. Menna, T. J.; Filisko, F. E. J Polym Sci Part B: Polym Phys 2004, 42, 4116-4125.

24. Figueiredo Neto, A. M. University of Sao Paulo, Sao Paulo, Brazil. Personal communication, 2003.

25. Larson, R. G. University of Michigan, Ann Arbor, MI. Personal communication, 2003.

26. Rosenblatt, C. Case Western Reserve University, Cleveland, OH. Personal communication, 2003.

27. Martin, D. C. Polymer 2002, 43, 4421-4436. 